\title{
Indocyanine green angiography-guided management of Vogt-Koyanagi-Harada disease: differentiation between choroidal scars and active lesions
}

\author{
Pascal B. Knecht • Alessandro Mantovani • \\ Carl P. Herbort
}

Received: 12 November 2012/ Accepted: 8 December 2012/Published online: 1 January 2013

(C) Springer Science+Business Media Dordrecht 2012

\begin{abstract}
When following Vogt-Koyanagi-Harada disease $(\mathrm{VKH})$, indocyanine green angiography (ICGA) is crucial in the subacute and convalescent stages of the disease in order to detect subclinical choroiditis and prevent the development of 'sunset glow' fundus. Hypofluorescent dark dots (HDDs) indicate persisting granulomas in the choroid. However, probably as a result of the healing process of choroidal granulomas, stromal choroidal fibrosis can also be shown by HDDs. We present two cases where intravenous corticosteroid administration because of persistent HDDs led to resolution of lesions in one case while they persisted in the other case. We reviewed the medical history of two VKH patients.
\end{abstract}

P. B. Knecht · C. P. Herbort $(\square)$

Retinal and Inflammatory Eye Diseases, Centre for Ophthalmic Specialized Care (COS), Clinic Montchoisi,

Rue de la Grotte 6, 1003 Lausanne, Switzerland

e-mail: carl.herb@bluewin.ch

P. B. Knecht

e-mail: pascalknecht@gmx.ch

P. B. Knecht

Department of Ophthalmology, University Hospital

Zurich, Zurich, Switzerland

A. Mantovani

Eye Clinic, Department of Ophthalmology,

Hospital Valduce, Como, Italy

C. P. Herbort

University of Lausanne, Lausanne, Switzerland
Complete routine work-up for patients with posterior uveitis was performed. The charts were screened for the presence of HDDs by ICGA in the subacute and convalescent stages of the disease before and after administration of body weight-adapted pulse intravenous methylprednisolone (PIM). The evolution of HDDs was studied and compared in both patients. A female patient presented with a persistent bilateral granulomatous panuveitis compatible with VKH. Cerebrospinal fluid analysis had shown lymphocytic pleocytosis. At presentation, therapy consisted of oral prednisone $80 \mathrm{mg} /$ day. Prednisone was tapered down to $22 \mathrm{mg} /$ day over 3 months, when a recurrence occurred with the presence of disseminated HDDs. PIM was administered, followed by oral corticosteroids. After 8 days of therapy, ICGA showed an almost complete disappearance of HDDs. A girl presented with bilateral panuveitis and widespread depigmented areas of her fundus. Cerebrospinal fluid analysis showed monocytic pleocytosis. Because of relative resistance to oral inflammation suppressive therapy (IST), PIM was administered for 3 days. Nevertheless, ICGA showed persistence of HDDs. Therapy was continued, and 3 months later, a followup ICGA still depicted numerous HDDs. Another PIM course was given, which had no effect on ICGA signs. HDDs in this case were interpreted as stromal choroidal scars. ICGA-guided therapy (mainly HDD evolution monitoring) helps to eradicate occult stromal disease in VKH and avoids 'sunset glow' fundus, by allowing precise adjustment of therapy. In some 
cases, HDDs do not represent active lesions but presumed intrastromal scars which need to be identified. A limited course of maximal IST including PIM can unmask such cases and avoid overtreatment of these patients.

Keywords Vogt-Koyanagi-Harada disease · Indocyanine green angiography .

Disease activity · Uveitis

\section{Introduction}

Vogt-Koyanagi-Harada disease (VKH) is a systemic disease with bilateral ocular involvement caused by an interleukin-2 family-driven Th17 response against tyrosinase family proteins in melanocytes [1-3]. Since these antigens, in the posterior segment, are exclusively found in the choroidal stroma, VKH should be classified as a 'primary stromal choroiditis', because choroidal inflammation is specifically directed at elements of the choroidal stroma. Only when choroidal inflammation spills over into neighbouring structures, manifestations like multifocal exudative nonrhegmatogenous retinal detachments occur around the optic disc and in the posterior pole [4, 5]. Indocyanine green angiography (ICGA) is especially suited to monitor choroidal involvement because it can detect preclinical choroidal inflammatory lesions before they spread, as well as persistent choroidal inflammation after apparent clinical disease has been mastered $[6,7]$. The most important ICGA sign in VKH is clearly the occurrence of hypofluorescent dark dots (HDDs), a result of diffusion impairment of the ICG molecule in the choroidal stroma due to spaceoccupying lesions. They are best visible in the intermediate and late phases of the ICGA and indicate stromal granulomas as shown by histopathology [8]. Other reliable ICGA signs for evaluation and for follow-up include hyperfluorescent choroidal vessels, fuzzy indistinct large stromal vessels indicating choroidal vasculitis, late diffuse hyperfluorescence and, lastly, ICGA disc hyperfluorescence in cases of hyperacute disease. Those ICGA signs were seen in almost $100 \%$ of fresh, de novo, untreated cases [9]. In recurrent attacks the same signs can be observed. However in chronic smouldering disease only HDDs and fuzzy indistinct choroidal vessels indicate ongoing occult choroidal inflammation [10, 11]. During this stage, no other clinical signs appear. This subclinical evolution can very well explain the development of 'sunset glow' fundus despite (suboptimal) therapy. 'Sunset glow' fundus, still considered to be the natural course of the disease, is simply the result of insufficiently treated disease $[6,7,12,13]$.

When following VKH with the help of ICGA for optimal therapy in the subacute and convalescent stages of the disease and in order to prevent the development of 'sunset glow' fundus, HDDs are the essential parameter to allow optimal adjustments to treatment of subclinical disease.

HDDs usually represent active stromal choroidal granulomas; however, they can also image stromal choroidal fibrosis probably as a result of the healing process of choroidal granulomas. This work aims to show that active HDDs can be differentiated from scarred lesions.

\section{Methods}

We reviewed the medical history of two patients who were examined, treated and followed at the Centre for Ophthalmic Specialized Care (COS), Lausanne, Switzerland, between 1995 and 2012. Case 1 presented in August 1995 and Case 2 in November 2005, both being referred with sight-threatening posterior uveitis suspected to be due to VKH. Ocular examination included best-corrected visual acuity, intraocular pressure (IOP) measurement, grading of intraocular inflammation using both classical criteria as well as laser flare photometry [14, 15]. Optical coherence tomography, fluorescein angiography (FA), ICGA, and cerebrospinal fluid (CSF) analysis as well as auditory tests were performed when deemed appropriate. Both patients underwent complete laboratory routine work-up applied to patients with posterior uveitis. The study was performed in accordance with the declaration of Helsinki.

The charts were screened for HDDs in ICGA in the subacute stage of the disease before and after administration of body weight-adjusted pulse intravenous methylprednisolone (PIM; Solu-Medrol ${ }^{\circledR}$; Pfizer AG, Zurich, Switzerland). The evolution of HDDs was studied and compared in both patients. 


\section{Case reports}

Case 1

A female South American patient was referred at the age of 24 for a second opinion with a persistent bilateral granulomatous panuveitis compatible with VKH. Onset of uveitis had been diagnosed 3 months earlier in another ophthalmology department. CSF analysis had shown lymphocytic pleocytosis. No other systemic signs were found. Further investigations excluded infectious or neoplastic etiologies. At presentation in our center, the patient was receiving $80 \mathrm{mg}$ prednisone per day. Laser Flare Photometry (LFP) measured $8.0 \mathrm{ph} / \mathrm{ms}$ in the right eye and $6.0 \mathrm{ph} / \mathrm{ms}$ in the left eye. FA revealed optic disc hyperfluorescence, and ICGA demonstrated remaining disseminated HDDs. Corticosteroids were then slowly tapered. At a dosage of $22 \mathrm{mg} /$ day, inflammation flared up again leading to a granulomatous anterior uveitis, vitritis and peripheral exudative detachments. LFP showed increased values to $28.8 \mathrm{ph} / \mathrm{ms}$ in the right eye and $33.2 \mathrm{ph} / \mathrm{ms}$ in the left eye. ICGA showed multiple HDDs in the fundus, whereas FA did not show gross alterations (Fig. 1). PIM was administered $1 \mathrm{~g}$ /day for 4 days, followed by oral corticosteroids. Concomitantly, immunosuppressive therapy with cyclosporine A (Sandimmune $^{\circledR}$; Novartis, Basel, Switzerland) $5 \mathrm{mg} / \mathrm{kg}$ body weight per day was introduced. Eight days after starting this regime (4 days after PIM), ICGA showed almost complete disappearance of HDDs (Fig. 1). Prednisone was tapered to $7.5 \mathrm{mg}$ /day without a flare up for more than 1 year, when another recurrence occurred. During the follow-up, an aseptic necrosis of the femoral head occurred, leading to a hip replacement. This patient was left under prolonged immunosuppressive therapy including cyclosporine A and azathioprine (Imurek $^{\circledR}$; Pro Concepta, Zug, Switzerland) $2.3 \mathrm{mg} / \mathrm{kg}$ body weight per day, finally resulting in a quiet stage without HDDs 4 years after starting immunosuppressive therapy, without recurrence after discontinuation of therapy during a follow-up of 8 years, and could be considered to be 'healed'.

Case 2

A 16-year-old girl presented elsewhere with decreased vision on both sides, accompanied by headaches. Clinical examination revealed anterior chamber inflammation with granulomatous keratic precipitates, posterior synechiae, vitreous inflammation and optic disc hyperemia. IOP was $8 \mathrm{mmHg}$ in the right eye and $7 \mathrm{mmHg}$ in the left eye. Fundus examination showed diffuse bilateral depigmentation, compatible with 'sunset glow' fundus (Fig. 2). FA showed optic disc hyperfluorescence, faint, barely visible peripheral leakage, but no pinpoint lesions (leakage) at the posterior pole. On ICGA, multiple HDDs could be seen at the posterior pole and mid-periphery indicating stromal choroidal granulomas. Lumbar puncture showed CSF pleocytosis with increased intracranial pressure. Further investigations excluded infectious or neoplastic etiologies.

At referral to our center 6 weeks after presentation elsewhere and 6 weeks after starting oral corticosteroids $(50 \mathrm{mg} / \mathrm{day})$, LFP measured $38.9 \mathrm{ph} / \mathrm{ms}$ in the right eye and $84.6 \mathrm{ph} / \mathrm{ms}$ in the left eye. Ultrasound biomicroscopy showed widespread supracilliary effusion associated with acute myopisation ( -2.0 diopters). Since there was no improvement after several days of oral corticosteroids, PIM was administered $500 \mathrm{mg} /$ day for 3 days. Concomitantly, azathioprine $2.3 \mathrm{mg} / \mathrm{kg}$ body weight was started. After PIM, another ICGA showed numerous persisting HDDs. Therapy was continued, and a follow-up ICGA was performed after 3 months (Fig. 3). Numerous HDDs could still be seen at the identical locations compared with prior examinations, and the question arose whether these lesions were active or might represent choroidal scars. Another PIM course was given, which had virtually no effect on ICGA signs (Fig. 3). The failure of HDDs to respond to PIM together with the presence of hypopigmented fundus lesions strongly suggested cicatricial lesions in the choroidal stroma. Unless new HDDs were identified on subsequent angiographies, which was not the case, ICGA findings in this case should not be interpreted as persistence of choroiditis and should not be taken in account to justify increase of therapy unlike in Case 1 and in most cases of $\mathrm{VKH}$, where persistence of HDDs means persistence of active subclinical choroiditis.

\section{Discussion}

The two principal difficulties in the treatment of VKH patients are on one hand the deferred initiation of therapy because of diagnostic delay and on the other 
Fig. 1 Case1:

Hypofluorescent dark dots (HDDs) in indocyanine green angiography (ICGA) caused by active disease. Persisting HDDs in a treated case of Vogt-KoyanagiHarada disease (ICGA fluorescein angiographic (FA) frame (FA before) only showing diffuse

irregularities at the level of the retinal pigment

epithelium. Four days after a 3 -day course of intravenous methylprednisolone (500 $\mathrm{mg}$ /day) most of the HDDs have disappeared or are only faintly visible (ICGA after); please note the restoration to a quasi normal aspect of the fuzzy appearance of the choroidal vessels before treatment. No significant post-treatment FA changes (FA after) before) with corresponding
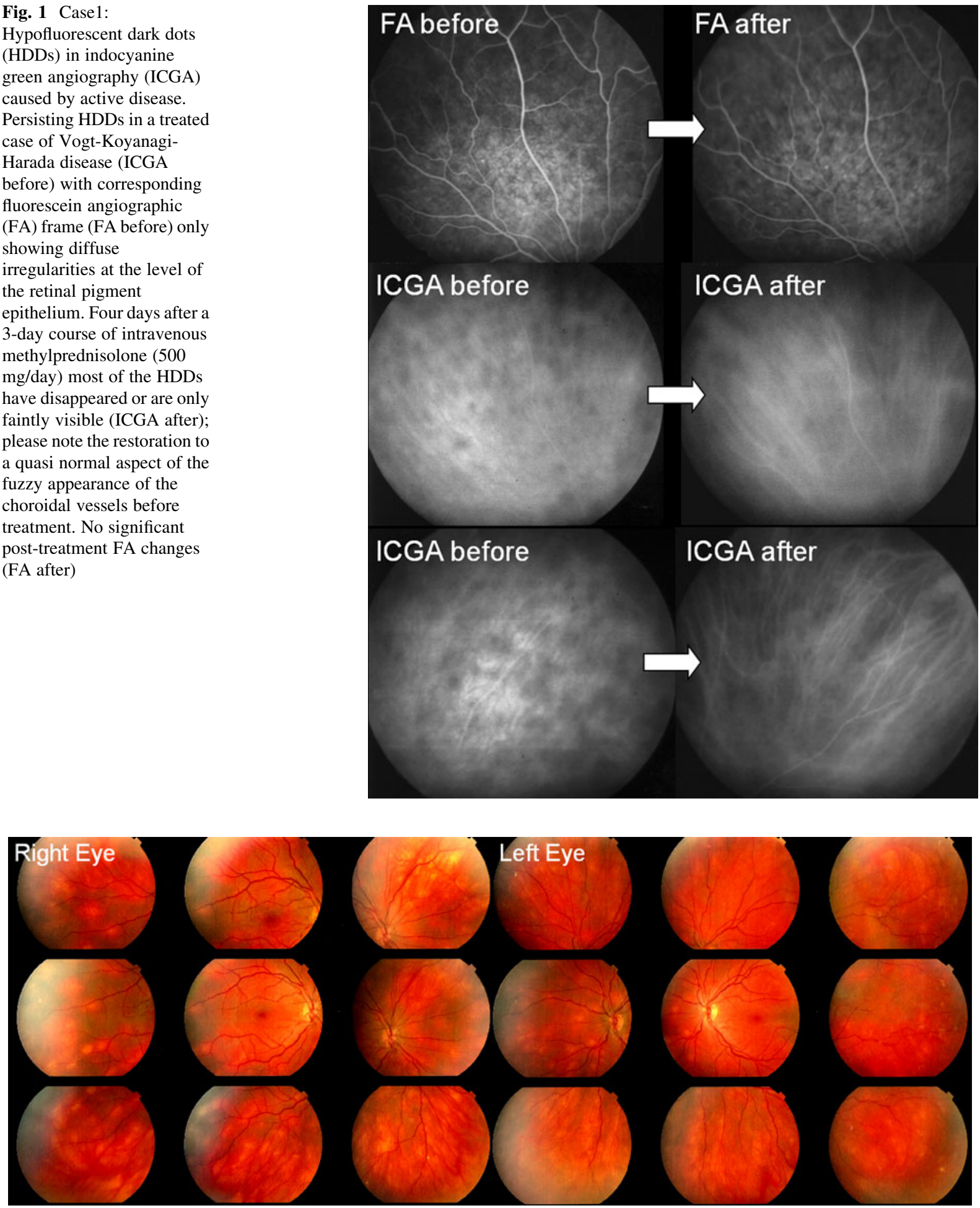

Fig. 2 Fundus photography of Case 2 suffering from VKH, showing "sunset glow" fundus in both eyes 
Fig. 3 Case 2: Persistence of hypofluorescent dark dots (HDDs) in indocyanine green angiography (ICGA) after intravenous methylprednisolone identifies HDDs as choroidal scars rather than active lesions. The fluorescein angiographic (FA) frames show no change before and after intravenous methylprednisolone therapy. The ICGA frames show that only some macular HDDs resolve after intravenous methylprednisolone therapy, indicating that all other HDDs correspond to choroidal scars. Images are obtained with the Staurenghi 230 SLO Retina Lens

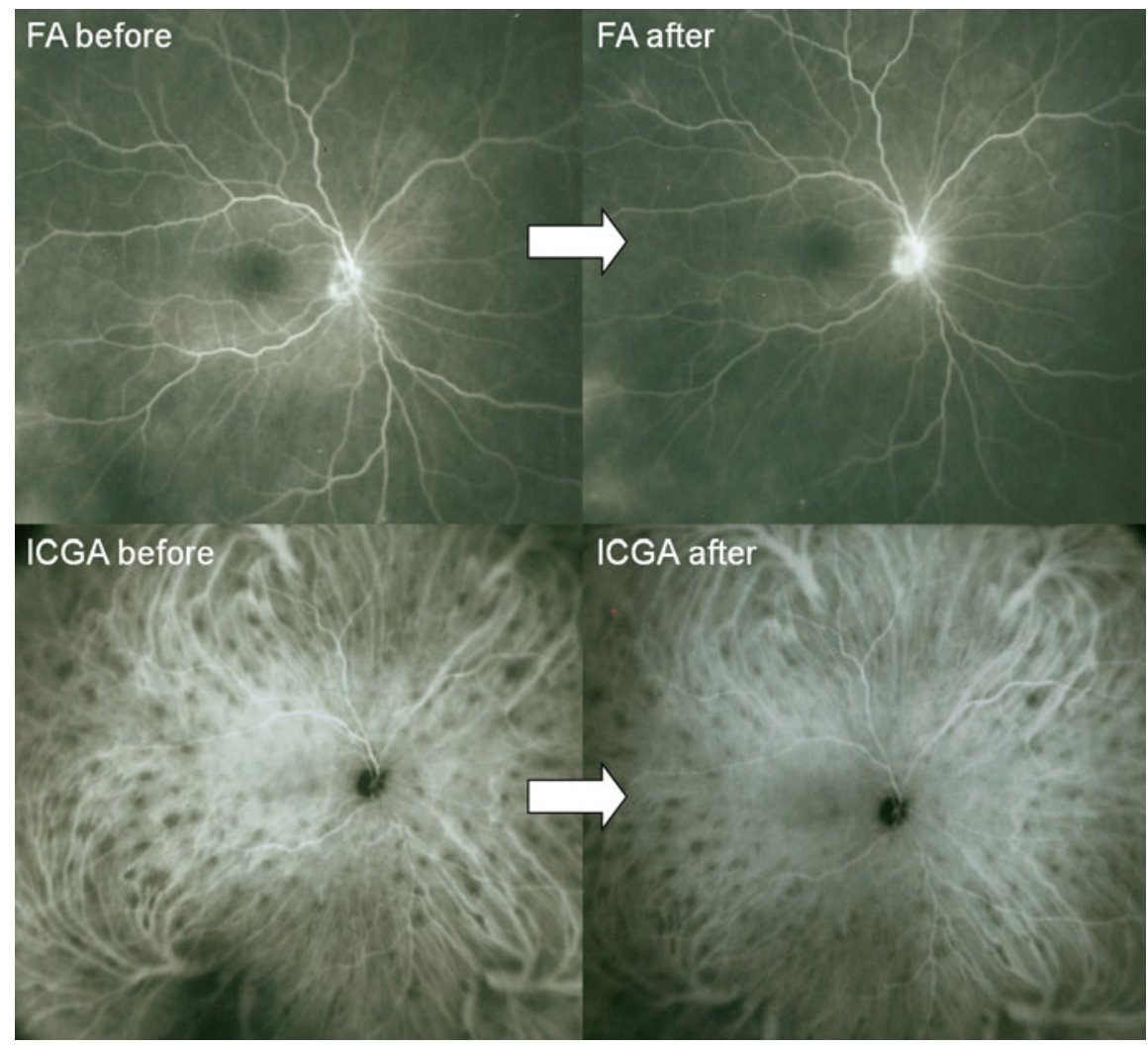

hand the classical treatment strategy put forward in most textbooks and publications that is purely based on the evolution of the clinically apparent extrachoroidal disease [16]. The treatment regimen published and practiced for $\mathrm{VKH}$ is now known to be insufficient as far as dose, duration and addition of immunosuppressants are concerned [13, 16-18]. In our opinion, VKH therapy should include the following three phases-(1) treatment of the uveitic acute exudative stage of the disease, (2) monitoring of the resolution of choroidal inflammation with the help of ICGA in the subacute stage of the disease (first 4 months of disease), and (3) ICGA-assisted tapering of therapy with re-increase of therapy at each subclinical ICGA-detected recurrence of choroidal inflammation during post-acute stages.

As long as a minimal threshold corticosteroid therapy is used in the acute phase, the type of treatment (with or without PIM) could not be shown to determine the final outcome [17]. The disease stages for which adequate treatment is of utmost importance are (2) the subacute stage when extra-choroidal disease is under control and regression of occult choroidal lesions is occurring and (3) the subsequent convalescent stage when occult recrudescence of choroiditis can occur at a time when IST is very low or has been discontinued [18]. ICGA monitoring for these phases has been published previously [19].

During these stages, clinical signs are mostly absent in insufficiently treated VKH patients, while subclinical choroiditis is progressing, resulting in 'sunset glow' fundus in almost $100 \%$ of cases This occult smouldering disease can be detected by ICGA and 'cured' in a large proportion of patients if treatment is adjusted accordingly. This is more often the case in those patients treated early, i.e., a few days to a few weeks after the onset of an inaugural inflammatory episode [6].

The only way to monitor occult choroiditis is the use of ICGA and in order to optimize treatment, ICGA-guided management has been proposed [6, 19]. The principal ICGA signs used to monitor therapy are HDDs. In the majority of cases, HDDs indicate active choroiditis. In rare instances, however, HDDs can be the result of choroidal scarring. If this is suspected, in cases of failure of HDDs to resolve despite adequately dosed IST, it is important to identify the cicatricial character of these ICGA lesions in order not to 
overtreat patients. Very often the resolution of all other ICGA signs such as fuzziness of choroidal vessel aid in diagnosing the absence of choroiditis despite the presence of HDDs, but sometimes reinstitution of maximal therapy is necessary to test whether or not HDDs resolve.

Administration of high-dose PIM for diagnostic purposes might raise the question of safety. Severe adverse events are reported, like sight-threatening rise of IOP [20], infections [21], bone loss [22] or even sudden death due to cardiovascular events [23]. However, in all reports it is underscored that these events are rare [24, 25]. For our purpose, PIM is sometimes justified since this is a valuable approach to treat recurrence of the disease. In case of choroidal scarring, the long-term benefit of not overtreating patients certainly supersedes the possibility of adverse events due to treatment with PIM.

In conclusion, ICGA-guided therapy helps to eradicate occult stromal disease in VKH and may avoid 'sunset glow' fundus, by allowing precise adjustment of therapy. In some cases HDDs do not represent active lesions but presumed intrastromal scars - a fact which needs to be identified. Maximal IST including PIM allows identification of such cases and avoids overtreatment of these patients.

Acknowledgments This work has been supported by the "Grieshaber Consulting" grant as well as the "Herta Messerli" trust.

\section{References}

1. Wang C, Tian Y, Lei B et al (2012) Decreased IL-27 expression in association with an increased Th17 response in Vogt-Koyanagi-Harada disease. Invest Ophthalmol Vis Sci 53:4668-4675

2. Yang Y, Xiao X, Li F et al (2012) Increased IL-7 expression in Vogt-Koyanagi-Harada disease. Invest Ophthalmol Vis Sci 53:1012-1017

3. Yamaki K, Gocho K, Hayakawa K, Kondo I, Sakuragi S (2000) Tyrosinase family proteins are antigens specific to Vogt-Koyanagi-Harada disease. J Immunol 165:73237329

4. Nussenblatt R, Whitcup S (2010) Uveitis, 4th edn. Mosby Elsevier, Philadelphia

5. Yanoff M, Duker JS (2009) Ophthalmology, 3rd edn. Mosby Elsevier, Philadelphia

6. Bouchenaki N, Herbort CP (2011) Indocyanine green angiography guided management of Vogt-KoyanagiHarada disease. J Ophthalmic Vis Res 6:241-248
7. Herbort CP, Mantovani A, Bouchenaki N (2007) Indocyanine green angiography in Vogt-Koyanagi-Harada disease: angiographic signs and utility in patient follow-up. Int Ophthalmol 27:173-182

8. Inomata H, Sakamoto T (1990) Immunohistochemical studies of Vogt-Koyanagi-Harada disease with sunset sky fundus. Curr Eye Res 9(Suppl):35-40

9. Miyanaga M, Kawaguchi T, Miyata K et al (2010) Indocyanine green angiography findings in initial acute pretreatment Vogt-Koyanagi-Harada disease in Japanese patients. Jpn J Ophthalmol 54:377-382

10. Okada AA, Mizusawa T, Sakai J, Usui M (1998) Videofunduscopy and videoangiography using the scanning laser ophthalmoscope in Vogt-Koyanagi-Harada syndrome. Br J Ophthalmol 82:1175-1181

11. Yuzawa M, Kawamura A, Matsui M (1993) Indocyanine green video-angiographic findings in Harada's disease. Jpn J Ophthalmol 37:456-466

12. Nakao K, Abematsu N, Mizushima Y, Sakamoto T (2012) Optic disc swelling in Vogt-Koyanagi-Harada disease. Invest Ophthalmol Vis Sci 53:1917-1922

13. Suzuki S (1999) Quantitative evaluation of "sunset glow" fundus in Vogt-Koyanagi-Harada disease. Jpn J Ophthalmol 43:327-333

14. Hogan MJ, Kimura SJ, Thygeson P (1959) Signs and symptoms of uveitis I. Anterior uveitis. Am J Ophthalmol 47:155-170

15. Herbort CP, Guex-Crosier Y, de Ancos E, Pittet N (1997) Use of laser flare photometry to assess and monitor inflammation in uveitis. Ophthalmology 104:64-71; discussion 71-62

16. Abu El-Asrar AM, Hemachandran S, Al-Mezaine HS, Kangave D, Al-Muammar AM (2012) The outcomes of mycophenolate mofetil therapy combined with systemic corticosteroids in acute uveitis associated with VogtKoyanagi-Harada disease. Acta Ophthalmol 90(8):e603-e608

17. Read RW, Yu F, Accorinti M et al (2006) Evaluation of the effect on outcomes of the route of administration of corticosteroids in acute Vogt-Koyanagi-Harada disease. Am J Ophthalmol 142:119-124

18. Kawaguchi T, Horie S, Bouchenaki N et al (2010) Suboptimal therapy controls clinically apparent disease but not subclinical progression of Vogt-Koyanagi-Harada disease. Int Ophthalmol 30:41-50

19. Bouchenaki N, Herbort CP (2001) The contribution of indocyanine green angiography to the appraisal and management of Vogt-Koyanagi-Harada disease. Ophthalmology 108:54-64

20. Gupta SR, Suhler EB, Rosenbaum JT (2010) Intravenous methylprednisolone can cause an acute, vision-threatening rise in intraocular pressure. J Clin Rheumatol 16:397-398

21. Badsha H, Edwards CJ (2003) Intravenous pulses of methylprednisolone for systemic lupus erythematosus. Semin Arthritis Rheum 32:370-377

22. Haugeberg G, Griffiths B, Sokoll KB, Emery P (2004) Bone loss in patients treated with pulses of methylprednisolone is not negligible: a short term prospective observational study. Ann Rheum Dis 63:940-944

23. Erstad BL (1989) Severe cardiovascular adverse effects in association with acute, high-dose corticosteroid administration. DICP 23:1019-1023 
24. Markomichelakis NN, Halkiadakis I, PapaeythymiouOrchan $S$ et al (2006) Intravenous pulse methylprednisolone therapy for acute treatment of serpiginous choroiditis. Ocul Immunol Inflamm 14:29-33
25. Bartalena L, Krassas GE, Wiersinga W et al (2012) Efficacy and safety of three different cumulative doses of intravenous methylprednisolone for moderate to severe and active graves' orbitopathy. J Clin Endocrinol Metab 97(12):4454-4463 